\title{
Michel Beniamino, Lise Gauvin (sous la direction de), Vocabulaire des études francophones. Les concepts de base
}

\section{Alessandro Corio}

\section{(2) OpenEdition \\ 1 Journals}

\section{Edizione digitale}

URL: https://journals.openedition.org/studifrancesi/46325

DOI: $10.4000 /$ studifrancesi. 46325

ISSN: 2421-5856

\section{Editore}

Rosenberg \& Sellier

\section{Edizione cartacea}

Data di pubblicazione: 1 octobre 2007

Paginazione: 479-480

ISSN: 0039-2944

\section{Notizia bibliografica digitale}

Alessandro Corio, «Michel Beniamino, Lise Gauvin (sous la direction de), Vocabulaire des études francophones. Les concepts de base», Studi Francesi [Online], 152 (LI | II) | 2007, online dal 30 novembre 2015, consultato il 24 novembre 2021. URL: http://journals.openedition.org/studifrancesi/46325 ; DOI: https://doi.org/10.4000/studifrancesi.46325

Questo documento è stato generato automaticamente il 24 novembre 2021.

\section{cc) (†) $\odot$}

Studi Francesi è distribuita con Licenza Creative Commons Attribuzione - Non commerciale - Non opere derivate 4.0 Internazionale. 


\title{
Michel Beniamino, Lise Gauvin (sous la direction de), Vocabulaire des études francophones. Les concepts de base
}

\author{
Alessandro Corio
}

\section{NOTIZIA}

MICHEL BENIAMINO, LISE GAUVIN (sous la direction de), Vocabulaire des études francophones. Les concepts de base, Limoges, Presses Universitaires de Limoges (Pulim), 2005, pp. 210.

Dalla "a" di "Aire Culturelle", alla "x" di "Xenologie", questo vocabolario di studi francofoni si rivela uno strumento davvero utile e ben elaborato sia a livello della ricerca specialistica e dello studio delle letterature e delle culture francofone, sia per l'insegnamento e l'apprendimento nella scuola secondaria e all'università. Come notano i curatori nell'Avant-Propos, l'estrema rarità di pubblicazioni a carattere pedagogico concernenti le letterature francofone evidenzia chiaramente i limiti della teorizzazione in questo campo di ricerca e la loro marginalizzazione nel sistema scolastico e universitario. Se a ciò si affianca la "giovinezza" che caratterizza la disciplina e la sua tradizione di insegnamento, si rende evidente la necessità dell'elaborazione di uno strumento di lavoro di questo genere. Trattandosi comunque di una canonizzazione, per quanto parziale, di autori e di concetti, un dizionario comporta anche dei rischi notevoli, di cui i curatori del volume e delle singole voci sembrano ben consapevoli. A un'esigenza di chiarezza e di semplicità, si affianca quindi il tentativo di costruire non una semplice presentazione della materia e dei suoi concetti già consolidati, ma anche del dibattito critico e delle sfaccettature che li contraddistinguono all'interno di un universo, quello della francofonia, che si caratterizza proprio per la sua eterogeneità e diversità, geografica, culturale, generazionale, ecc. Il rischio è sempre quello di solidificare nozioni, concetti, 
tradizioni, scuole di pensiero producendo una normalizzazione disciplinare e una sclerosi intellettuale che risulti miope nei confronti dei continui cambiamenti, dibattiti interni ed esterni alla disciplina stessa, ambivalenze ed evoluzioni teoriche, linguistiche, letterarie. Se l'insegnamento si concepisce infatti non come "indottrinamento", bensì come continua rinegoziazione dei concetti e delle prospettive critiche che tenga conto della loro ricezione, spesso sfaccettata e diversificata, uno strumento di questo genere deve favorire e spingere verso un approccio autonomo e critico di studenti, insegnanti e ricercatori ai soggetti che affrontano. Un obiettivo che pare ben raggiunto dai curatori e dai numerosi collaboratori che hanno compilato le voci di questo vocabolario e che sono tra i maggiori studiosi mondiali di letterature francofone. Ne citiamo solamente alcuni: Dominique Chancé (Bordeaux III), Jacques Chevrier (Paris IV), Lieven D'Hulst (Leuven), Daniel Delas (Cergy Pontoise), Gilles Dupuis (Montréal), Jean-Louis Joubert (Paris XIII), Josias Semujanga (Montréal) e ovviamente i due curatori, Lise Gauvin (Montréal) e Michel Beniamino (Limoges).

Ci risulta impossibile, chiaramente, rendere conto qui della totalità delle voci e della loro stratificazione e interazione, segnalata per altro chiaramente da un apparato di rimandi infratestuali tra le varie voci, e da una ricca e ben documentata intertestualità, fatta di riferimenti, citazioni scelte in modo calibrato e accurato e, soprattutto, da una bibliografia essenziale relativa alle singole voci che segnala le opere letterarie e critiche fondamentali, fornendo un utile strumento senza risultare pesante né invadente. Tra le voci scelte, relativamente poche ma ben approfondite, ritroviamo quindi una serie di concetti essenziali all'interno del dibattito francofono, quali "Altérité", "Bilinguisme", "Créolisation", “Créolité", "Dialogisme”, "Francophonie", "Hybridité”, "Identité culturelle", "Négritude", "Oraliture", "Postcolonialisme", ecc., ma anche termini meno noti, più specifici o attinenti ai margini di un'area disciplinare che si rivela capace di dialogare con l'antropologia, la linguistica, la filosofia, ecc.: "Aire culturelle", "Ethnotexte”, “Épilinguistique”, “Exote”, "Folklorisation”, “Idiolecte”, “Mythe”, "Nation", "Norme", ecc.

3 A titolo di esempio, scegliamo una voce curata da Dominique Chancé, Créolisation (pp. 49-54). L'estensione del commento e dell'analisi rivela subito che non ci troviamo di fronte a una secca definizione di un concetto sedimentato, bensì a una genealogia critica che ne sottolinea le varie fasi di elaborazione all'interno dell'opera di Édouard Glissant, nonché le sue origini e i suoi riferimenti ad altri autori e critici, il successo della sua ricezione anche in altre discipline come l'antropologia e la critica letteraria comparatista, nonché le critiche e i limiti del concetto e le sue ulteriori evoluzioni (vedi la voce successiva: Créolité). La voce è così suddivisa in quattro sottosezioni i cui titoli sono esplicativi del gioco dialettico e del movimento di differenza (si tratterebbe qui di una différance alla Derrida) da cui si è generato il concetto stesso, segnalato dalla barra tra "Antillanité/créolisation", "Créolisation/créolité", "Créolisation/mondialisation" e "Créolisation/Chaos-monde". Oltre a far riferimento ad altri scrittori caraibici che si sono avvicinati a questa nozione, quali Alejo Carpentier (Cuba), Wilson Harris (Guyana) e Edward Kamau Brathwaite (Jamaïque), e al paradosso che assegna un'origine linguistica al termine, poi esteso a un ambito più vasto di tipo socio-culturale. Dominique Chancé analizza accuratamente la genealogia del concetto nell'opera di Glissant, a partire dal Discours antillais (1981) sino alle sue opere più recenti, Poétique de la Relation (1990) e Traité du Tout-monde (1997), nonché il dibattito cui ha dato origine con la generazione successiva degli scrittori della créolité, a partire dall'éloge de la 
créolité (Chamoiseau, Confiant, Bernabé, 1993) e le prese di distanza dello stesso Glissant dalle possibili derive identitarie a cui è stato sottoposto. Mettendo infine in relazione questo termine che, più che un concetto, vuole indicare un "immaginario" in continua mutazione, coi fenomeni complessi della globalizzazione e con le sue forme di rappresentazione letteraria, come il "neobarocco", o con le sue teorizzazioni critiche e linguistiche, Chancé conclude segnalando l'esigenza di un'analisi e di un approfondimento dei suoi processi specifici, delle sue dinamiche e delle sue condizioni, non solo nel contesto caraibico, ma anche nelle sue diversificate riterritorializzazioni globali.

4 Questo Vocabolario, appassionante e ben curato, si rivela pertanto uno strumento prezioso per gli specialisti, ma anche per coloro che vogliono avvicinarsi a un campo letterario e a un dibattito critico che rivela sempre di più la sua ricchezza e la sua attinenza nei confronti di fenomeni culturali, sociali e politici planetari. 